\title{
Multi Contact Points Slider for A Surface Acoustic Wave Motor
}

\author{
Member Minoru Kurosawa, non-Member Makoto Chiba, \\ and Member Toshiro Higuchi (The University of Tokyo)
}

Key words: Micro actuator, Ultrasonic motor, Surface acoustic wave, Piezoelectric actuator, Friction drive

\section{Introduction}

The operation of a friction drive surface acoustic wave motor has been demonstrated(1). For friction drive at high frequency vibration, the high contact pressure such as several hundreds MPa is required. The operation conditions and the basic performances have been investigated in experiments(2). From our recent research, to obtain the large output force, there were two important points(2).(3). First, pressing force, namely, pre-load should be adjusted that the depression of slider and stator was the half of the vibration displacement. Second, the contact area of the slider and the stator should be enlarged to make good use of the large thrust density.

To enlarge the contact area, the multi contact points slider was made on trial and the output force became $25 \mathrm{mN}(3)$. But it was only 10 percent of the estimated maximum driving force of $0.3 \mathrm{~N}$. In this study, we have improved the way of making the multi contact points slider and pressing the slider. The experiment was carried out to investigate the driving availability of the multi contact points slider.

\section{Multi Contact Points Slider}

In order to obtain the large contact area, a flat contact surface slider on a flat surface substrate is quite common. From a tribological point of view, however, it is almost impossible to make the flat surface slider contact to the stator due to the squeeze film pressure. The multi contact points slider was made of a $\mathrm{LiNbO}_{3}$ piezoelectric plate and about 500 steel balls. The steel balls were glued on the plate

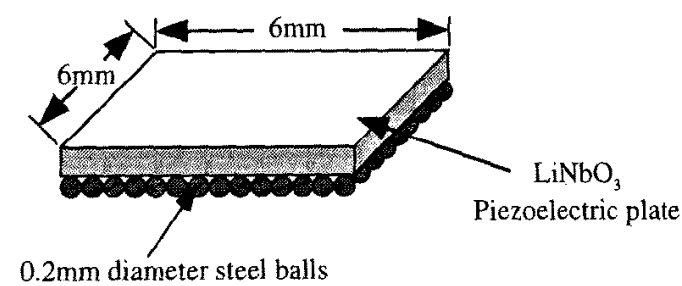

Fig.1 Multi contact points slider. as illustrated in Fig.1. The surface of $\mathrm{LiNbO}_{3}$ was mirror finished, so that the roughness was order of several $\mathrm{nm}$. The steel balls were glued using elastic adhesive. Owing to the adhesive elasticity, the contact points became in a plane and automatically adjusted in spite of the tolerance of the diameter of the steel balls.

For the wide contact area and the higher pre-load operation, the diameter of the steel balls should be small(3). A diameter of the steel balls glued on the $\mathrm{LiNbO}_{3}$ plate was $0.2 \mathrm{~mm}$ because they were obtained easily. The amount of the steel balls was about 500 .

\section{Experiment Using Multi Contact Points Slider}

The experiment was carried out using the swing arm type surface acoustic wave motor as shown in Fig. 2. Using this apparatus, the linear motion of the multi contact points slider was changed to the swing motion of the beam. The swing angle of the beam was measured using a rotary encoder. The multi contact points slider was pressed by a coil spring placed in the spring housing. The pre-load was changed from $300 \mathrm{mN}$ to $2400 \mathrm{mN}$.

The driving frequency was $9.6 \mathrm{MHz}$ and the voltage was $100 \mathrm{~V}_{\mathrm{o}-\mathrm{p}}$. The optimum depression is half of the vibration displacement, namely about $9 \mathrm{~nm}$ in this case. The optimum pre-load was calculated to be $2.7 \mathrm{mN}$ for each steel ball, and $1400 \mathrm{mN}$ for the slider. The whole contact area of the slider was $2.5 \times 10^{-3} \mathrm{~mm}^{2}$, namely about $1 / 10000$ of the plate area. The maximum contact pressure was 800 MPa at the center of each contact point. From the pre-load of $1.4 \mathrm{~N}$ and the friction coefficient of 0.2 , estimated driving force became about $0.3 \mathrm{~N}$.

Figure 3 shows the output force as a function of the pre-load. The output force increased up to the optimum pre-load of $1400 \mathrm{mN}$. On the other hand, beyond the optimum pre-load condition, the output force decreased. In this experiment, the maximum driving force was $0.3 \mathrm{~N}$ when the pre-load was about $1400 \mathrm{mN}$. These values were almost same as those of calculated values.

From the thrust density of $120 \mathrm{~N} / \mathrm{mm}^{2}$, the maximum 


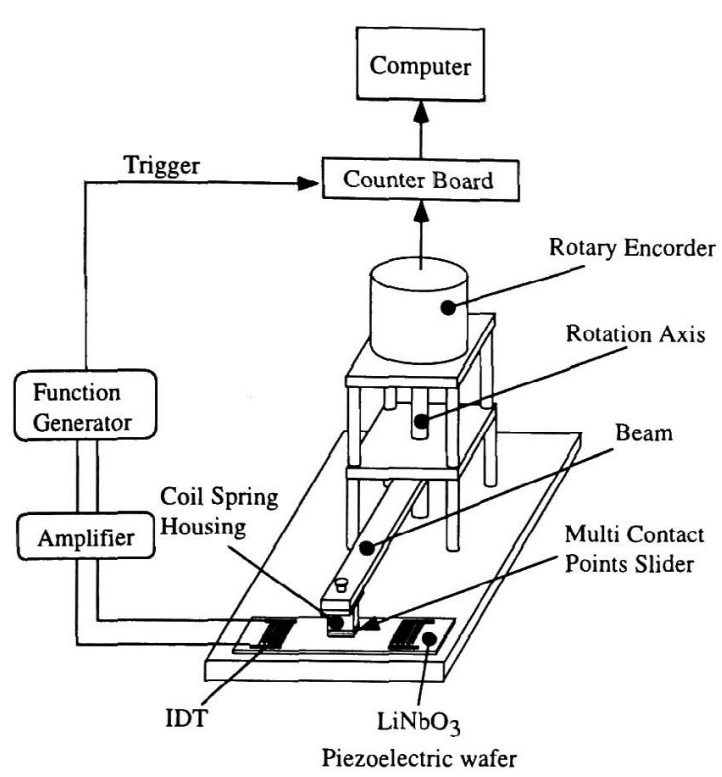

Fig. 2 Swing arm type surface acoustic wave motor: $\mathrm{LiNbO}_{3}$ stator transducer is $15 \times 60 \times 1 \mathrm{~mm}^{3}$.

driving force of $0.3 \mathrm{~N}$ was obtained. It is predicted that the driving force will become higher, if the contact area is enlarged. When the contact area becomes 10 times as large as that of this experiment, the driving force will become $3 \mathrm{~N}$. In this case, the contact area is only $0.1 \%$ that of the slider area.

\section{Conclusion}

We have succeeded in improvement for friction drive conditions of the surface acoustic wave motor. To enlarge the contact area and the pre-load, the multi contact points slider was made on trial. As a result the driving force became $0.3 \mathrm{~N}$. It was 300 times as large as that of the single steel ball slider result of $0.95 \mathrm{mN}$. Because of the huge thrust density such as $120 \mathrm{~N} / \mathrm{mm}^{2}$, the multi contact points slider has an ability to make the driving force be much higher up to several newton.

\section{Acknowledgment}

This work was supported by the Grant-in-aid for general scientific research of the Ministry of Education, Science, Sports and Culture.

(Received March 10, 1997)

\section{References}

(1) M. Kurosawa, M. Takahashi and T. Higuchi, "Ultrasonic linear motor using surface acoustic waves", IEEE Trans. on Ultrasonics, Ferroelectrics, and Frequency control, 43, 5, 901-906, 1996.

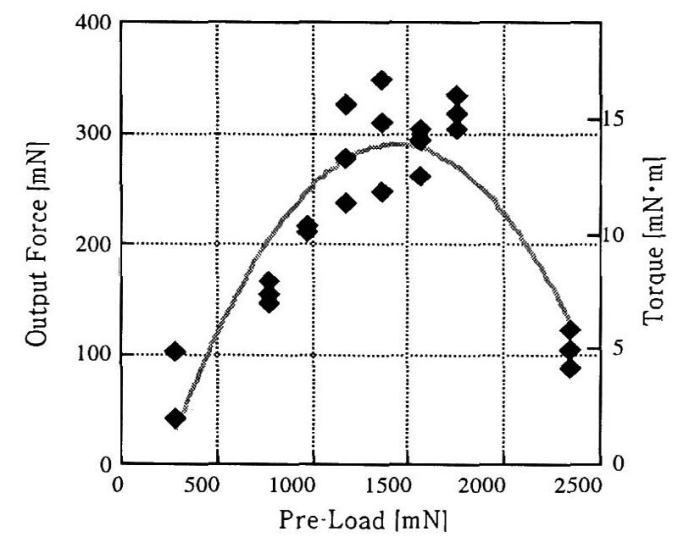

Fig.3 Output force as a function of the pre-load.

(2) M. Kurosawa, M. Takahashi, and T. Higuchi, "Optimum Pre-Load of Surface Acoustic Wave Motor", Proc. Of IEEE International Ultrasonics Symp. '96, San Antonio, Texas, (Nov. 3-6, 1996).

(3) M. Chiba, M. Takahashi, M. Kurosawa, and T. Higuchi, "Evaluation of a surface acoustic wave motor output force", Proc. of IEEE Workshop on MEMS, Nagoya, Japan, 250-255(1997).

Minoru Kurosawa (formerly Kuribayashi) (Member)

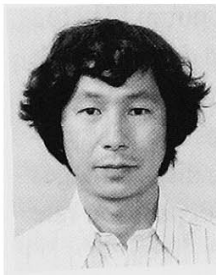
was born in Nagano, Japan, on April 24, 1959. He received the B. Eng. degree in electrical and electronic engineering, and the M. Eng. and D. Eng. degrees from Tokyo Institute of Technology in 1982, 1984, and 1990. Since 1992, he has been an Associate Professor at the Graduate School of Engineering, The University of Tokyo.

Makoto Chiba (non-Member) received the B. Eng.

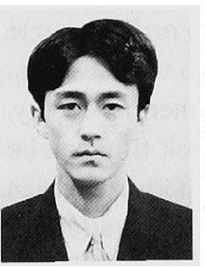
degree in precision machinery engineering from the University of Tokyo in 1995. Since 1995, he has been with the Graduate School of Engineering, The University of Tokyo.

Toshiro Higuchi (Member) was born in Ehime, Japan on

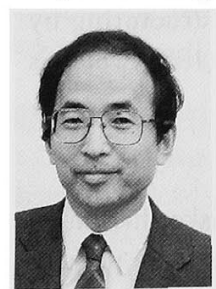
February 26, 1950. He received B.E., M.S., and $\mathrm{Ph} . \mathrm{D}$.degrees in precision machinery engineering from The University of Tokyo in 1972, 1974, and 1977, respectively. Since 1991, he has been a Professor at the Graduate School of Engineering, The University of Tokyo. 